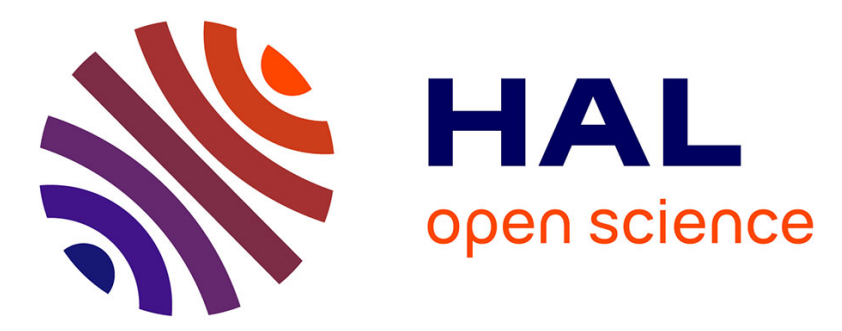

\title{
Updating 3D acoustic models with the constitutive relation error method: A two-stage approach for absorbing material characterization
}

\author{
V. Decouvreur, Pierre Ladevèze, Philippe Bouillard
}

\section{- To cite this version:}

V. Decouvreur, Pierre Ladevèze, Philippe Bouillard. Updating 3D acoustic models with the constitutive relation error method: A two-stage approach for absorbing material characterization. Journal of Sound and Vibration, 2008, 310 (4-5), pp.985-997. 10.1016/j.jsv.2007.08.012 . hal-01648332

\author{
HAL Id: hal-01648332 \\ https://hal.science/hal-01648332
}

Submitted on 13 Jan 2020

HAL is a multi-disciplinary open access archive for the deposit and dissemination of scientific research documents, whether they are published or not. The documents may come from teaching and research institutions in France or abroad, or from public or private research centers.
L'archive ouverte pluridisciplinaire HAL, est destinée au dépôt et à la diffusion de documents scientifiques de niveau recherche, publiés ou non, émanant des établissements d'enseignement et de recherche français ou étrangers, des laboratoires publics ou privés.

\section{(c)(1)}

Distributed under a Creative Commons Attribution| 4.0 International License 


\title{
Updating 3D acoustic models with the constitutive relation error method: A two-stage approach for absorbing material characterization
}

\author{
V. Decouvreur ${ }^{\mathrm{a}, 1}, \mathrm{P}$. Ladevèze ${ }^{\mathrm{b}}, \mathrm{Ph}$. Bouillard ${ }^{\mathrm{a}, *}$ \\ ${ }^{\mathrm{a}}$ BATir (Building, Architecture and Town planning) Department, Université Libre de Bruxelles, 50 av. F. D. Roosevelt CP 194/2, \\ 1050 Brussels, Belgium \\ ${ }^{\mathrm{b}}$ Laboratoire de Mécanique et Technologie, ENS-Cachan Université Paris VI/CNRS, 61 av. Président Wilson, 94235 Cachan, France
}

\begin{abstract}
In the global framework of improving vibro-acoustic numerical simulations together with the need to decrease the number of prototyping stages, improving the quality for acoustic models becomes increasingly important for many industries such as automotive companies, for instance. This paper focuses on achieving greater accuracy for acoustic numerical simulations by making use of a parametric updating technique, which enables tuning the model parameters inside physically meaningful boundaries. The improved model is used for the next prototyping stages, allowing more accurate results within reduced simulation times. The updating technique used in this paper is based on recent works dealing with the constitutive relation error (CRE) method applied to acoustics. The updating process focuses on improving the acoustic damping matrix related to the absorbing properties of the materials covering the borders of the acoustic domain.

The present study proposes a 2-stage optimization process, which exhibits many advantages. Indeed, the computational time decreases, the frequency interpolation of the material absorbing properties outside the studied frequency range is easily performed, and comparing the correlation of several material absorbing constitutive equations with experimental records is fast.

Additional originality of the work comes with the application of the CRE updating method to a concrete real-life device, while previous works addressed purely numerical setups without experimental data. The test-case is the TRICARMO setup engineered by LMS International in Leuven, Belgium. The TRICARMO setup is a simplified car cabin with rigid walls and car seats inside. Thanks to the 2-stage approach, the material property characterization of the seat is improved by running the updating simulation process using a physical absorbing material model.
\end{abstract}

*Corresponding author. Tel.: + 35281992609

E-mail address: Philippe.Bouillard@ulb.ac.be (Ph. Bouillard).

${ }^{1}$ Research fellow supported by the F.R.I.A. foundation 


\section{Introduction}

In recent years, many industries have taken into account noise control consideration during the design stages, either to satisfy code rules or to improve the end-user's comfort. To evaluate and further improve the numerical model, experimental information remains highly useful.

Updating techniques aim at getting numerically simulated physical fields that are closer to the measured ones. Among these techniques, some act on tuning model parameters inside physically meaningful boundaries. Such methods allow to use the new parameters in further computations while changing the configuration.

In Ref. [1], the choice of using the constitutive relation error (CRE) method to update acoustic models is motivated, and the principles of the CRE are extensively explained. The technique is also applied to 2D academic test-cases. The good results encouraged the authors to apply the CRE technique to 3D real-life test cases, but the developed technique showed a drawback in terms of computational time. Indeed, the CRE updating technique requires solving a matrix system that is larger than the initial one. Furthermore, the technique being iterative, several hundred inversions of the larger matrix are performed for each frequency. One of the goals achieved by the 2-stage approach presented here is to decrease the running time.

To face real-life test cases, the admittance model that is used to evaluate the absorbing properties of the porous materials has to be as accurate as possible. The present paper uses Wilson's admittance formulation [2] to characterize the porous compounds of the numerical applications.

The clue of the updating technique presented here is to split the updating process into a first stage that has to be independent of any admittance model, and a second stage that links the microstructural material properties to the material absorbing behavior through an admittance model, as explained in Section 2. In the first stage, each absorbing material is characterized by a single complex number for each updating frequency.

In the second stage, a physical admittance model is used to fit as well as possible the real and imaginary parts of the admittance coefficients that have been found thanks to the first stage. This fitting stage is processed through the studied frequency range for each absorbing material.

With the goal to compare several admittance models when updating an acoustic setup, the frequency correlation with the updated admittance coefficients using a specific absorption model during the second stage is treated like a post-treatment analysis and is very cheap in terms of CPU time.

Mathematical foundation of the CRE updating method is formulated in Section 1.1. The wall admittance modeling is described in Section 1.2, and Section 1.2.3 presents the admittance model of Wilson that will be used later to update the numerical applications treated in the article.

The 2-stage approach detailed description together with a validation study are parts of Section 2 .

Section 3 addresses the updating of a real-life device: a concrete simplified car engineered by LMS International, which is called TRICARMO. Car seats are placed into the setup and are the only absorbing facilities damping the acoustic waves inside the TRICARMO car. The setup is instrumented with many microphones recording the sound pressure level inside the car.

The 2-stage updating technique is used to update the driver's seat absorbing properties through a frequency range that goes from 50 to $350 \mathrm{~Hz}$.

Finally, Section 4 presents the conclusions about the ability of the 2-stage updating approach to accurately improve the numerical simulation of acoustic absorbing properties.

\subsection{Updating acoustic models with the CRE}

The present paper uses a parametric updating technique based on the CRE. The fundamentals of the CRE were first developed by Ladevèze in structural dynamics (see Ref. [3]) and then applied to acoustics in Ref. [1] The main idea in the CRE technique consists in splitting the data and equations of the model into 'reliable' information and 'less reliable' one. Whether one trusts a given data or equation has to be related to the assumptions made in its derivation.

\subsubsection{The reliable information}

A solution has to verify the reliable information exactly. In what concerns acoustics, it means that the pressure field $p$ has to satisfy the Helmholtz wave equation with the associated Dirichlet and Neumann 
boundary conditions on parts $\partial_{1} \Omega$ and $\partial_{2} \Omega$ of the boundary, respectively:

$$
\left\{\begin{array}{l}
\text { Helmholtz }: \Delta p+k^{2} p=0, \\
\text { Dirichlet B.C. }: p_{\left.\right|_{\mathrm{\partial}_{1} \Omega}}=\bar{p}, \\
\text { Neumann B.C. }: v_{n_{\left.\right|_{2} \Omega}}=\left.\frac{\mathrm{j}}{\omega \rho} \frac{\partial p}{\partial n}\right|_{\mathrm{\partial}_{2} \Omega}=\bar{v}_{n},
\end{array}\right.
$$

where $\mathrm{j}=\sqrt{-1}, \omega$ is the angular frequency, $k$ is the wavenumber, $\rho$ is the fluid density, $v_{n}$ is the normal velocity, and $\bar{p}$ and $\bar{v}_{n}$ are the imposed pressure and normal velocity on parts $\partial_{1} \Omega$ and $\partial_{2} \Omega$ of the boundary, respectively.

\subsubsection{The less reliable information}

The best candidate among a solution set satisfying the reliable information minimizes the CRE estimator, which is built on the less reliable information.

In particular, through this paper, it is chosen that the Robin admittance boundary condition together with the measured pressure field amplitude will not be verified exactly, assuming that those data are the most questionable for the present acoustic model. Practically, the pressure field $q$ will satisfy the Robin equation (2), and the distance between pressure variables $p$ and $q$ will be minimized:

$$
\text { Mixed Robin B.C. : } v_{\left.\right|_{\left.\right|_{3} \Omega}}=A_{n}(\omega) q \text {, }
$$

where $A_{n}$ is the admittance coefficient, and $\partial_{3} \Omega$ defines the absorbing boundary where Eq. (2) applies. As to the uncertainty on the measured pressure levels, discrepancies between computed pressure $p$ and measured field $\tilde{p}$ has to be minimized also.

The two contributions to the less reliable information result in building the error estimator CRE:

$$
e_{\omega}^{2}=\omega^{2} \rho^{2} \int_{\partial_{3} \Omega}\left(v_{n}-A_{n} q\right)^{*}\left(v_{n}-A_{n} q\right) \mathrm{d} \Gamma+\frac{r}{1-r}\|p-\tilde{p}\|^{2},
$$

where $v_{n}=(\mathrm{j} / \omega \rho)(\partial p / \partial n)$ and $(.)^{*}$ denotes the complex conjugate. The weighting factor $r /(1-r)$ is related to the confidence in the measurements in comparison with the model accuracy. In the absence of a priori knowledge in the measurement quality, $r$ is set to 0.5 , keeping in mind that $0 \leqslant r<1$.

\subsubsection{Discrete formulation of the CRE updating method}

The continuous acoustic model can be approximated by a discrete formulation, e.g. using the finite element formalism based on a variational form of reliable Eqs. (1) and (2). Updating the approximated discrete model is achieved by solving the finite element matrix equation while minimizing the CRE estimator, as presented in Eq. (4).

$$
\text { Find } s_{\omega}=(\mathbf{P}, \mathbf{Q}) \mid\left\{\begin{array}{l}
{[\mathbf{K}] \mathbf{P}+\mathrm{j} \omega \rho[\mathbf{C}] \mathbf{Q}-\omega^{2}[\mathbf{M}] \mathbf{P}=[\mathbf{E}] \mathbf{P},} \\
e_{\omega}^{2}\left(s_{\omega}\right) \text { is minimum, }
\end{array}\right.
$$

where the discrete form of the modified CRE (3) taking into account the experimental error is given by

$$
e_{\omega}^{2}=\omega^{2} \rho^{2}(\mathbf{Q}-\mathbf{P})^{*}[\mathbf{D}](\mathbf{Q}-\mathbf{P})+\frac{r}{1-r}(\mathbf{\Pi P}-\tilde{\mathbf{P}})^{*}\left[\mathbf{G}_{\mathbf{w}}\right](\mathbf{\Pi P}-\tilde{\mathbf{P}}) .
$$

The definition of the finite-element vectors and matrices is as follows:

- $p^{h}=\mathbf{N}^{t} \mathbf{P}$ and $q^{h}=\mathbf{N}^{t} \mathbf{Q}$ are approximated pressure fields ( $\mathbf{N}$ being the shape functions),

- $[\mathbf{M}]=\frac{1}{c^{2}} \int_{\Omega} \mathbf{N}^{t} \mathbf{N} \mathrm{d} \Omega$ is the mass matrix ( $c$ being the sound speed),

- $[\mathbf{K}]=\int_{\Omega}^{c^{2}} \boldsymbol{\nabla}^{t} \mathbf{N} \boldsymbol{\nabla N} \mathrm{N} \Omega$ is the stiffness matrix,

- $[\mathbf{C}]=\int_{\partial_{3} \Omega} A_{n} \mathbf{N}^{t} \mathbf{N} \mathrm{d} \Gamma$ is the admittance matrix,

- $[\mathbf{E}]=\int_{\partial_{2} \Omega}^{\partial_{2} \Omega} \nabla_{n}^{t} \mathbf{N N} \mathrm{d} \Gamma$ is the system excitation matrix due to normal velocities prescribed on boundary $\partial_{2} \Omega$,

- $[\mathbf{D}]=\int_{\partial_{3} \Omega} A_{n}^{*} A_{n} \mathbf{N}^{t} \mathbf{N} \mathrm{d} \Gamma$. 
$\left[\mathbf{G}_{\mathbf{w}}\right]$ represents the error measure $\|.\|^{2}$ of Eq. (3), $\boldsymbol{\Pi}$ is a projection operator that gives the value of the pressure at the corresponding sensors, and $\tilde{\mathbf{P}}$ is the nodal value vector of the measured pressure $\tilde{p}$.

In the numerical example of this paper, $\left[\mathbf{G}_{\mathbf{w}}\right]$ is a square unity matrix of size equal to the number of measurements.

The updating process is iterative: each step consists in computing new updating parameters and solving the problem using these new values. The computed pressure is compared to the measurements using the CRE, and the iterative process stops when the error is below a reference threshold value.

\subsection{Modeling of the wall admittance}

The sound absorption in porous media consists of 3 different contributions [4]:

- the viscous effects in the boundary layer close to the frame,

- the thermal diffusion process between the fluid and the frame,

- the internal energy losses coming from the frame motion.

The main geometrical parameters of these absorbent materials are:

- the porosity $\Omega=V_{f} / V_{t}$, where $V_{f}$ and $V_{t}$ are the fluid volume and the total volume, respectively,

- the tortuosity $q$,

- the average pore diameter $d(\mathrm{~m})$,

- the flow resistivity $\sigma\left(\mathrm{Nm}^{-4} \mathrm{~s}\right)$.

\subsubsection{Absorbing media and equivalent fluid}

The modeling of the absorbing media is based on the concept of equivalent fluid (see Ref. [4]). The heterogeneous porous medium is made of a skeleton/frame perforated by pores with various shape. Though, it is regarded as an homogeneous fluid, which is characterized by a complex propagation constant $\Gamma$, a complex impedance $Z_{c}$ and a thickness $d_{e}$ (see Fig. 1). The local phenomena occurring inside the absorbing material are not modeled. An acoustic wave entering the material is supposed to be reflected and to exit the material at the incidence location. This assumption is perfect for waves entering the medium perpendicularly to its surface. The outgoing acoustic wave is computed from the incident wave and the wall impedance representing the absorbing medium. If the incident wave enters the absorbing medium perpendicularly to its surface, the wall admittance $Z_{n}$ is given by Eq. (6).

$$
Z_{n}=Z_{c} \frac{Z_{c}-\left(\mathrm{j} Z_{n 0} / \tan \left(\Gamma d_{e}\right)\right)}{Z_{n 0}-\left(\mathrm{j} Z_{c} / \tan \left(\Gamma d_{e}\right)\right)}
$$

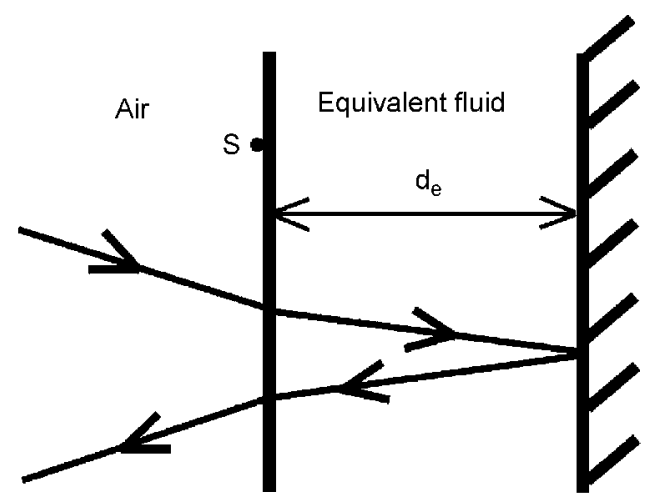

Fig. 1. Layer of equivalent fluid (thickness $d_{e}$ ) representing an absorbing material backed by a rigid impervious wall. 
where $Z_{n 0}$ is the wall admittance of the surface backing the absorbing material. In the particular case where the layer of equivalent fluid is backed by a rigid impervious wall, $Z_{n 0} \rightarrow \infty$, and Eq. (6) reduces to Eq. (7).

$$
Z_{n}=\frac{-\mathrm{j} Z_{c}}{\tan \left(\Gamma d_{e}\right)} .
$$

\subsubsection{Admittance models in the literature}

The admittance models presented in the literature can be split in 3 kinds of models:

- the empirical models,

- the microstructural models,

- the phenomenological models.

The microstructural models are derived by calculating the exact solution for the propagation of sound waves in pores of constant circular cross section. The resulting equations are tuned to accommodate more complicated geometries using one or two shape factors. While microstructural models provide a quite accurate absorption description for a broad range of frequencies and materials, their equations are very complicated to handle and require many experimental data to be provided.

Phenomenological models propose a compromise between microstructural and empirical models: they are derived from simplified propagation models inside porous media. They provide relations for $\Gamma$ and $Z_{c}$ that usually describe the absorbent materials nearly as well as microstructural models do. Hence, phenomenological models are easier to handle and less experimental information is required. Additional information about admittance models can be found in the literature of Ref. [2].

\subsubsection{Wilson's model}

Wilson's model is particularly interesting in the framework of updating updating materials because it can be described by 1, 4 or 5 parameters, and the model is able to mimic the frequency behavior of any empirical or microstructural admittance model. Wilson's equations describe the propagation of sound in porous materials as follows [2]:

$$
\begin{aligned}
& \left.\left.\frac{Z_{c}}{\rho c}=\frac{q}{\Omega}\left[1+\frac{\gamma-1}{\sqrt{1-\mathrm{j} \omega \tau_{\mathrm{ent}}}}\right) 1-\frac{1}{\sqrt{1-\mathrm{j} \omega \tau_{\mathrm{vor}}}}\right)\right]^{-1 / 2}, \\
& \left.\left.\frac{\Gamma}{\omega / c}=q\left[1+\frac{\gamma-1}{\sqrt{1-\mathrm{j} \omega \tau_{\mathrm{ent}}}}\right) / 1-\frac{1}{\sqrt{1-\mathrm{j} \omega \tau_{\mathrm{vor}}}}\right)\right]^{1 / 2},
\end{aligned}
$$

where $\rho$ is the fluid density, $c$ is the sound velocity, $\gamma$ is the specific heat ratio, $q$ is the tortuosity, $\Omega$ is the porosity, and $\sigma$ is the flow resistivity, an intrinsic property of the material that measures the resistance to an air flow through it. $\sigma$ lies between 5000 and $10^{5} \mathrm{Nm}^{-4} \mathrm{~s}$ for materials like fiberglass and open-bubble foam.

$\tau_{\text {ent }}$ is the relaxation time (i.e. the time necessary to return to an equilibrium state after a perturbation is introduced) related to the temperature gradient between the fluid and the frame. $\tau_{\mathrm{vor}}$ is the relaxation time of the pressure gradient related to the fact that the fluid sticks to the frame.

By default, Wilson's model exhibits 4 parameters which are $q, \Omega, \tau_{\text {ent }}$, and $\tau_{\text {vor }}$. With $X=\rho f / \sigma$ being the reduced frequency and $f=\omega / 2 \pi$ the frequency, the model of Wilson supposes to evaluate $\tau_{\text {ent }}$ and $\tau_{\text {vor }}$ at low and high reduced frequencies, let say at $X=0.01$ and 1 . Interpolating between these reduced frequency values gives the characteristic impedance and the propagation constant at mid frequencies. If one is interested in a given microstructural model, it is possible to mimic its behavior by computing $\tau_{\text {ent }}$ and $\tau_{\text {vor }}$ by making use of the equations for $Z_{c}(8)$ and $\Gamma(9)$.

In the particular case of the well known Delany-Bazley model [5], it is assumed that $\Omega=1$ and $q=1$. This is actually in good agreement with that simple model. Since this empirical model exhibits only one material dependent parameter, $\tau_{\text {ent }}$ and $\tau_{\text {vor }}$ are computed at the average reduced frequency $X=0.1$. The system of 2 equations (8) and (9) with 2 unknowns then yields to relations (10) and (11), which are functions of the 
reduced frequency, and then of the flow resistivity only:

$$
\begin{aligned}
& \left.\left.\frac{Z_{c}}{\rho c}=\left[1+\frac{\gamma-1}{\sqrt{1-\mathrm{j} 19 X}}\right) 1-\frac{1}{\sqrt{1-\mathrm{j} 13 X}}\right)\right]^{-1 / 2}, \\
& \left.\left.\frac{\Gamma}{\omega / c}=\left[1+\frac{\gamma-1}{\sqrt{1-\mathrm{j} 19 X}}\right) / 1-\frac{1}{\sqrt{1-\mathrm{j} 13 X}}\right)\right]^{1 / 2} .
\end{aligned}
$$

In Section 3.3, one uses Wilson's model for which $\tau_{\text {ent }}$ and $\tau_{\mathrm{vor}}$ are computed by comparing relations (8) and (9) to the microstructural model of Biot-Allard [6] at low and high frequencies. The relaxational times solving the equalities are:

$$
\begin{aligned}
\tau_{\text {vor }} & =2 \frac{\rho q^{2}}{\Omega \sigma}, \\
\tau_{\text {ent }} & =N_{\operatorname{Pr}} S_{b}^{2} \tau_{\text {vor }},
\end{aligned}
$$

where $N_{\text {Pr }}$ is the Prandtl number. That version of Wilson's model needs 4 absorbent material dependent parameters like the initial one of Biot-Allard. These parameters are the porosity $\Omega$, the tortuosity $q$, the flow resistivity $\sigma$, and a shape factor $S_{b}$.

\section{The 2-stage approach}

\subsection{Principles}

The 2-stage updating method splits the process in 2 phases. Compared to the CRE technique presented in Section 1.1, the 2-stage approach solves the same system of Eq. (4). The main difference resides in the optimization loop aiming at minimizing the error estimator (5). In the former CRE updating technique, the optimization process uses a classical gradient based method to choose the next candidate for each admittance coefficient. The choice consists in evaluating the gradient of the error estimator (5) with respect to each parameter of the admittance coefficients to find the best optimization direction minimizing the CRE. The time needed to find the next best candidate from iteration $k$ to $k+1$ is proportional to the number of parameters characterizing each absorbing material.

The first stage of the new updating technique then reduces the running time by characterizing each admittance coefficient using only two variables: the real and imaginary parts. Indeed, most of the admittance models use at least four intrinsic parameters to determine the absorbing properties of a given porous material. Knowing also that increasing the number of optimization parameters makes the search of the optimum solution more difficult, reducing the size of the variable space yields to stabilizing the optimization process. The first stage is run at a few frequencies evenly spread within the studied frequency range.

Then, the second step is launched to get a continuous description of each admittance coefficient through the frequency range. Based on an admittance model that is parameterized in terms of frequency and intrinsic structural material properties, the second stage consists in finding the best combination of parameters that generates a smooth frequency interpolation of the discrete set of real and imaginary parts found during the first stage. The fitting process of the second stage is once again based on a gradient optimization technique. This stage is though much faster since the functional to be minimized is the distance between two admittance coefficients while during stage one, each evaluation of the error estimator requires solving the finite element system of equations. For each absorbing material, the second stage can be written as

$$
\text { Find } s=\left(\alpha_{m}\right)_{\mid(m=1, \ldots, M)} \mid \sum_{l=1}^{L}\left\|A_{n, l}^{u p}-A_{n, l}(\omega, s)\right\| \text { is minimum, }
$$

where:

- $\alpha_{m}$ are the intrinsic parameters of the admittance model with $m=1, \ldots, M$,

- $L$ is the number of updating frequencies, 
- $A_{n, l}^{\text {up }}$ is the updated complex admittance coefficient from stage 1 ,

- $A_{n, l}(\omega, s)$ is the admittance coefficient computed using a frequency dependent model based on structural parameters $\alpha_{m}$.

In summary, the first stage looks for the best complex admittance coefficient for each material of the setup at a given frequency. The introduction of a particular admittance model is achieved through the second stage, which fits the complex admittance coefficients that were calculated during the first updating stage through the frequency range of interest. The variable parameters of the admittance model are physical properties of the absorbing material and can vary inside plausible boundaries.

\subsection{Comparing the 2-stage approach versus the former CRE method}

A flowchart comparing the former CRE updating technique versus the 2-stage version is presented in Fig. 2. The darker boxes highlight the differences between the two CRE based updating technique process flows.

From top to bottom in Fig. 2, the first discrepancies concern the frequency increment $\Delta f$ between two updating frequencies. The 2-stage technique allows for larger frequency increments since a frequencydependent admittance model is used during the fitting step of the second stage.

The second darker box indicates the use of a second stage only for the new updating process flow.

The bottom dark boxes explain the differences in the gradient-based optimization process of the first stage as mentioned in Section 2.1: the former updating technique calculates partial derivatives of the functional with respect to structural parameters of the absorbing materials, while the 2-stage approach considers only the derivatives with respect to the real and imaginary parts of the admittance coefficients.

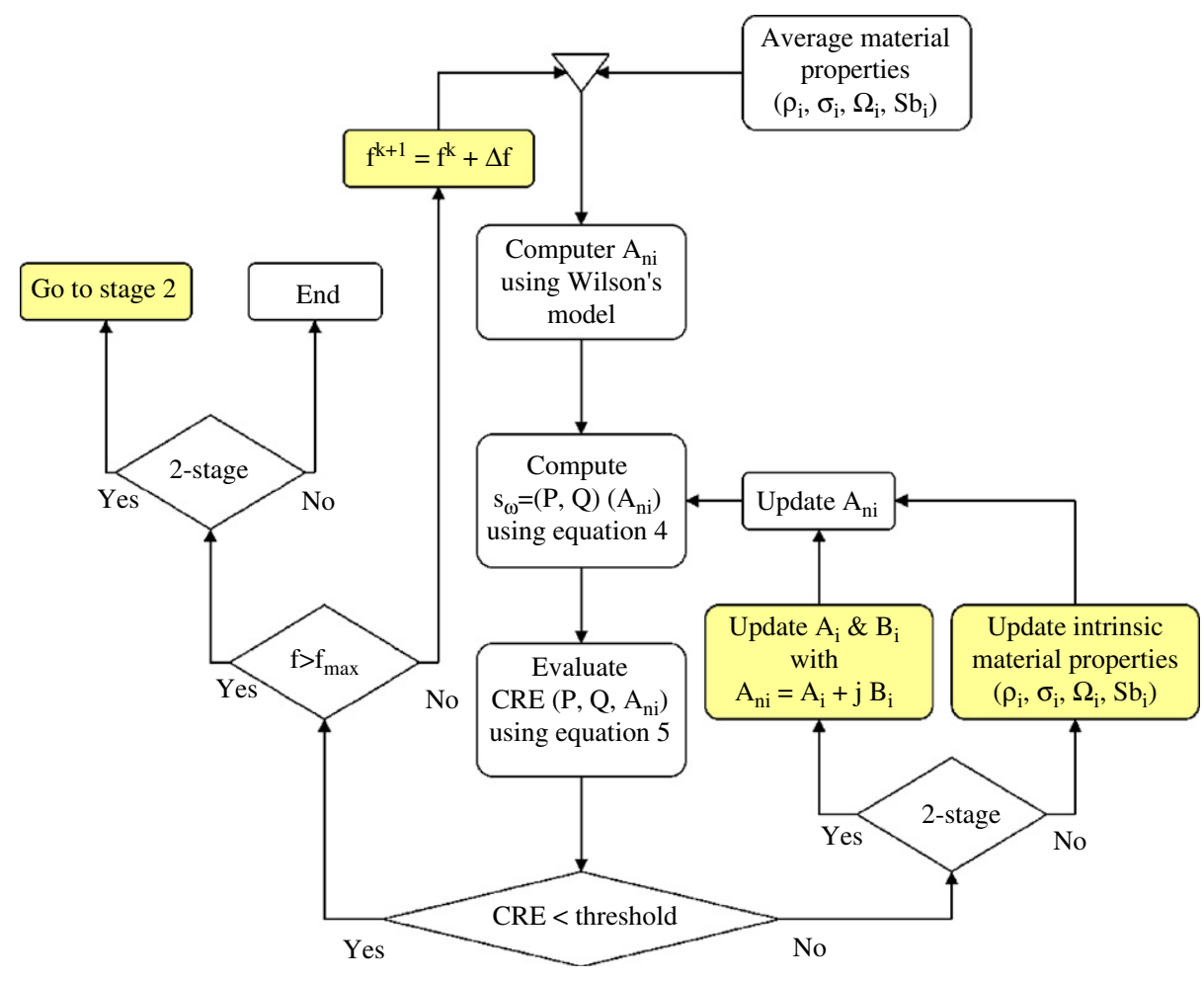

Fig. 2. Flowchart of the conventional CRE updating method versus the 2-stage approach. 


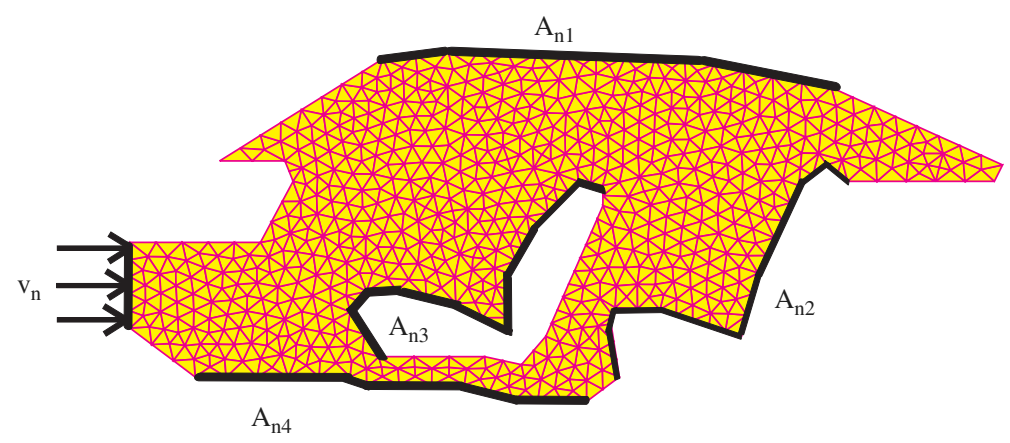

Fig. 3. Academic setup used to validate the 2-stage method : the car cabin is equipped with 4 absorbing materials $A_{n 1 \rightarrow 4}$ and the noise inside the acoustic domain is generated by the dashboard vibrations with normal velocity $v_{n}$.

\subsection{Validation of the 2-stage technique}

The goal of this part is to validate the 2-stage technique on a simple academic model inspired from Ref. [7]. The setup is a $2 \mathrm{D}$ car cabin that is covered with 4 different absorbing materials and excited by its firewall as described in Fig. 3. The absorption of the 4 layers is characterized using a 4 parameter Wilson's model with standard material properties for porous foam. The thickness of layers $1-4$ is $3,4,5$ and $6 \mathrm{~cm}$, respectively, and the corresponding material properties are initially determined to build a finite element reference pressure field that replaces the experimental data.

Then, the setup is updated to find back the right admittance properties, the initial values being voluntarily biased by about $30 \%$. The updating frequency range goes from 50 to $500 \mathrm{~Hz}$.

The device is firstly updated using a standard CRE method, which means using a 4 parameter description of the absorbing materials (Wilson's model) and a frequency increment of $10 \mathrm{~Hz}$ for instance.

Then, the 2-stage technique is applied to the academic device, updating the absorbing materials using its real and imaginary parts and a frequency increment of $40 \mathrm{~Hz}$. The fine frequency description of the admittance coefficients is obtained thanks to the second stage of the method that smoothes the discrete admittance values using its 4 parameter description.

On this simple example, the 2-stage technique runs 15 times quicker than the standard one. The quality of the results is examined in Fig. 4 by looking at the frequency plot of the 4 admittance coefficients provided by both updating techniques. The discrete values computed using the standard updating technique (tick marks in Fig. 4) and the smoothed interpolation calculated via the 2-stage approach (based on raw data that are not represented in Fig. 4) match perfectly, which validates the fast 2-stage method.

The microstructural parameters characterizing the absorbing materials that are used to build the reference pressure field are reported in Table 1. The same microstructural properties are used for all materials, the only changing parameter being the layer thickness, which is sufficient to change the admittance properties of the absorbing materials. The discrepancy between the reference parameters and the updated ones never reaches $1 \%$ no matter the updating technique, so that only reference values are presented in Table 1.

\section{Updating a real-life test case setup}

\subsection{TRICARMO setup description}

The TRICARMO setup is a simplified concrete car cabin that has been developed by LMS International in Leuven (see Fig. 5) in the framework of a research project founded by the Flemish Institute for the promotion of scientific and technological research in industry (IWT) [8]. The acoustic domain is excited by a loudspeaker that simulates the vibration of the dashboard, and a driver's seat lies in the car cabin. Experimental pressure fields to be used in the first updating stage are recorded in a few locations of the acoustic domain. The updating process aims at characterizing the absorbing properties of the seat. 

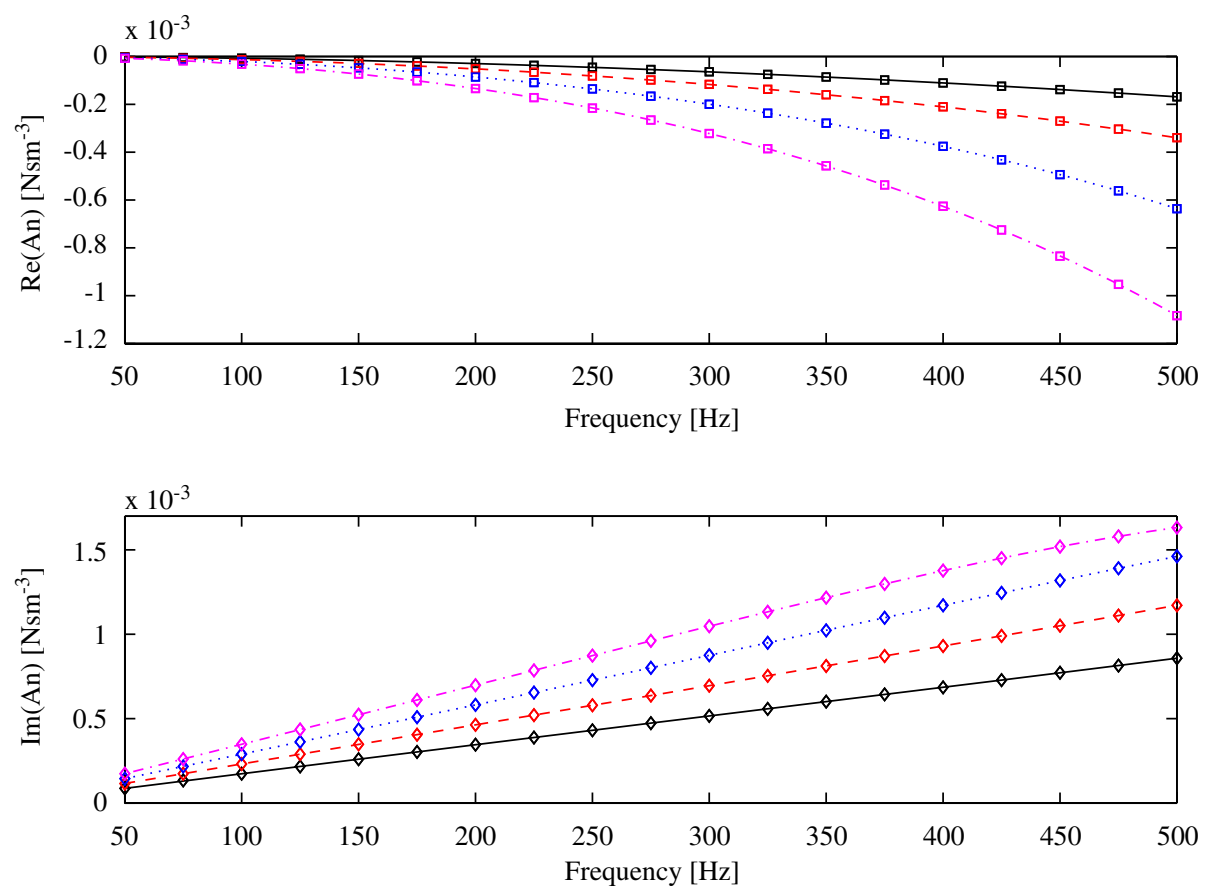

Fig. 4. Admittance coefficients computed with the standard discrete updating method (tick marks), and with the 2-stage approach (lines). Material 1, solid line; material 2, dashed line; material 3, dotted line; material 4, dash-dotted line.

Table 1

Microstructural parameters of the absorbing materials for the academic setup of Fig. 3

\begin{tabular}{lllll}
\hline $\begin{array}{l}\text { Admittance } \\
\text { coefficient }\end{array}$ & $\begin{array}{l}\text { Porosity } \\
\Omega\end{array}$ & $\begin{array}{l}\text { Tortuosity } \\
q\end{array}$ & $\begin{array}{l}\text { Flow resistivity } \\
\sigma\left(\mathrm{Nm}^{-4} \mathrm{~s}\right)\end{array}$ & $\begin{array}{l}\text { Shape factor } \\
S_{b}\end{array}$ \\
\hline$A_{n 1 \rightarrow 4}$ & 0.95 & 1 & 10,000 & 1 \\
\hline
\end{tabular}
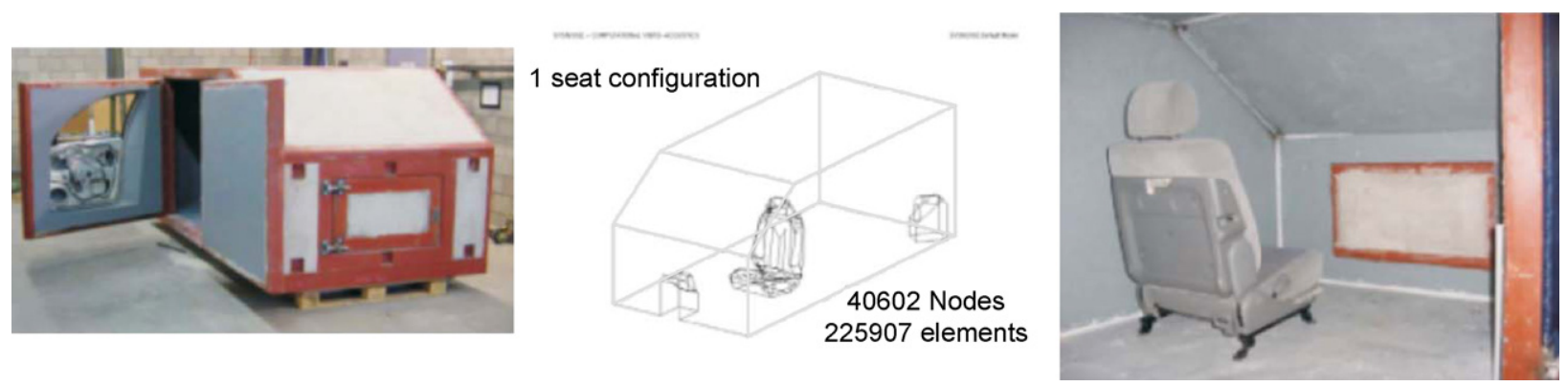

Fig. 5. TRICARMO concrete car with driver's seat (courtesy of LMS International).

\subsection{Driver's seat modeling}

The TRICARMO setup can be equipped with different seat configurations. While it is possible to achieve measurements with front and rear seats inside the concrete car, the results presented here focus on the "driver's seat only" configuration.

The seat modeling is split into a backrest and an horizontal cushion. Both cushions are represented by a two layer material. The bottom layer is a thick open cell foam (material $1 \mathrm{in} \mathrm{Fig.} \mathrm{6).} \mathrm{The} \mathrm{top} \mathrm{layer} \mathrm{consists} \mathrm{of} \mathrm{a} \mathrm{thin}$ 


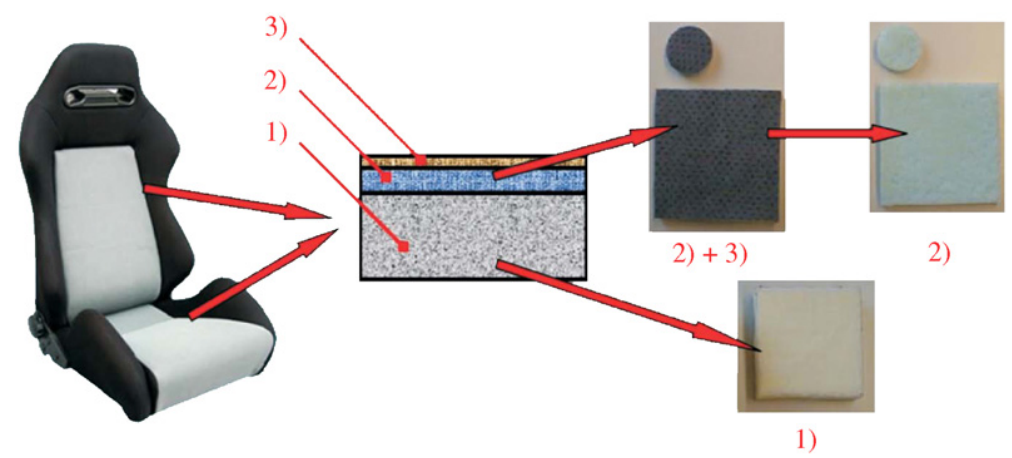

Fig. 6. Car seat analysis: backrest and bottom cushions are modeled by layers of porous materials: (1) thick open cell foam, (2) thin foam layer, (3) fabric skin (material sample pictures from Ref. [9]).

Table 2

Relative CPU time comparison for the different stages: stage time/total updating time

\begin{tabular}{llll}
\hline & Stage 1 & Stage 2 (Delany-Bazley) & Stage 2 (Wilson) \\
\hline CPU time (\%) & 98.1 & 0.8 & 1.1 \\
\hline
\end{tabular}

foam layer covered by a fabric skin (materials $2+3$ in Fig. 6). The cover fabric is not modeled separately and materials $2+3$ in Fig. 6 are merged together in one absorbing material for the numerical simulations. So, each seat cushion is regarded as the superposition of two absorbing materials with admittance coefficients $A_{n 1}$ and $A_{n(2+3)}$ and the equivalent wall admittance of the assembly is computed using Eq. (6).

The backrest and bottom cushion average thickness is, respectively, 60 and $55 \mathrm{~mm}$. In both cases, material $2+3$ accounts for $8.25 \mathrm{~mm}$ of the total thickness of the cushion. Samples of those materials were analyzed by the "Laboratorium voor Akoestiek en Thermische Fysika" of the Catholic University of Leuven (K.U.L.), Belgium. Material properties such as density, tortuosity, porosity, flow resistivity, etc., were evaluated to run deterministic numerical simulations with highly refined seat mesh in the framework of other studies. Some of these material properties will be used to check the updated results of the present analysis.

\subsection{Updating results}

The updating process looks for the best parameter combination to describe the frequency behavior of the absorbing materials of the seat. The updating is achieved through a frequency bandwidth of [50-350] $\mathrm{Hz}$ with a frequency step of $25 \mathrm{~Hz}$. Running the first stage provides the complex discrete admittance coefficients at frequencies $50,75,100, \ldots, 350 \mathrm{~Hz}$. Running the second stage then needs to choose an admittance model. For instance, this stage is run first with an empirical Delany-Bazley model, and then with a 4 parameter Wilson's phenomenological admittance model based on the Biot-Allard's microstructural one. Initial values for the parameters at the first iteration are set to $\Omega=1, q^{2}=1, \sigma=10^{4} \mathrm{Nm}^{-4} \mathrm{~s}$, and $S_{b}=1$. As discussed before, the second stage is very cheap in terms of CPU time compared to the first stage, as shown in Table 2 where the second stage typically accounts for about $1 \%$ of the total computational time. The entire updating process runs for $2 \mathrm{~h}$ on a single $2.4 \mathrm{GHz}$ Linux processor.

The second stage provides continuous values of the admittance coefficient from the discrete updated results. The smooth values are used to build the updated pressure field at the driver's ear in Fig. 7. The updated pressure/velocity ratio, which is called mobility, is plotted (actually its amplitude, in $\mathrm{dB}$ ) together with the experimental curve and the ratio computed without taking into account the absorbing properties of the seat (i.e. the undamped dotted FRF, Fig. 7). The updated FRF (dash-dotted line) fits rather well the measured one (solid line). This result could not necessarily have been foreseen since no information was provided for the porous material of the seat excepted its average thickness. Though, for this particular case, the slow frequency 


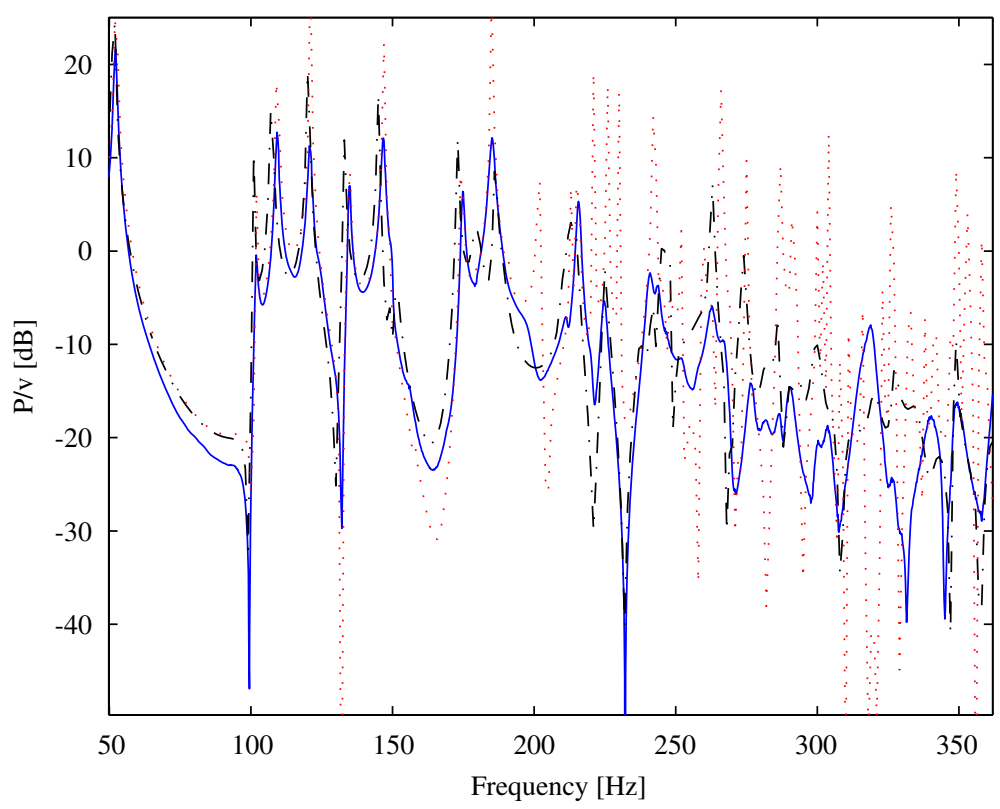

Fig. 7. Pressure/velocity ratio at the driver's ear: measured (solid line), computed without absorption from the seat (dotted line), and computed with updated absorption properties of the seat (dash-dotted line).
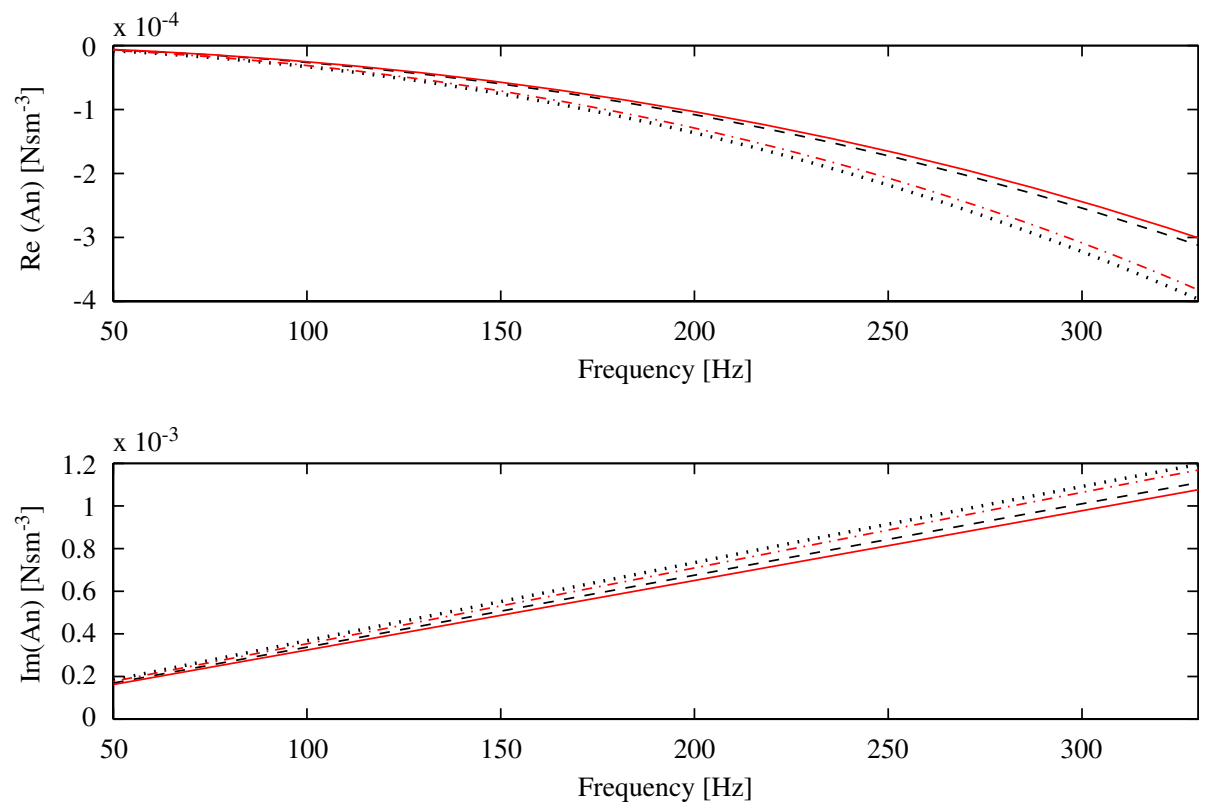

Fig. 8. Real and imaginary parts of the admittance coefficients of the backrest and bottom cushions constituting the seat computed with the measured and the updated microscopic properties. Measured bottom cushion, dashed line; updated bottom cushion, solid line; measured backrest cushion, dotted line; updated backrest cushion, dash-dotted line.

dependence of the homogenized parameters characterizing the porous media potentially improves the result quality of the method (see Fig. 8).

The updated material properties of the seat yield to admittance coefficients that are compared to the coefficients coming from the material properties measured in the lab. The comparison in Fig. 8 deals with the real and imaginary parts of the admittance coefficients of the backrest and bottom cushions of the seat. 
Measured versus updated microstructural parameters of the porous media

\begin{tabular}{|c|c|c|c|c|}
\hline & $\begin{array}{l}\text { Porosity } \\
\Omega\end{array}$ & $\begin{array}{l}\text { Tortuosity } \\
q\end{array}$ & $\begin{array}{l}\text { Flow resistivity } \\
\sigma\left(\mathrm{Nm}^{-4} \mathrm{~s}\right)\end{array}$ & $\begin{array}{l}\text { Shape factor } \\
S_{b}\end{array}$ \\
\hline$A_{n 1}$ (measured) & $>0.95$ & 1.28 & 9053 & - \\
\hline$A_{n(2+3)}$ (measured) & $>0.95$ & 1.16 & 7282 & - \\
\hline$A_{n 1}$ (updated) & 0.99 & 1.32 & 10,505 & 1.05 \\
\hline$A_{n(2+3)}$ (updated) & 0.99 & 1.19 & 7337 & 0.86 \\
\hline
\end{tabular}
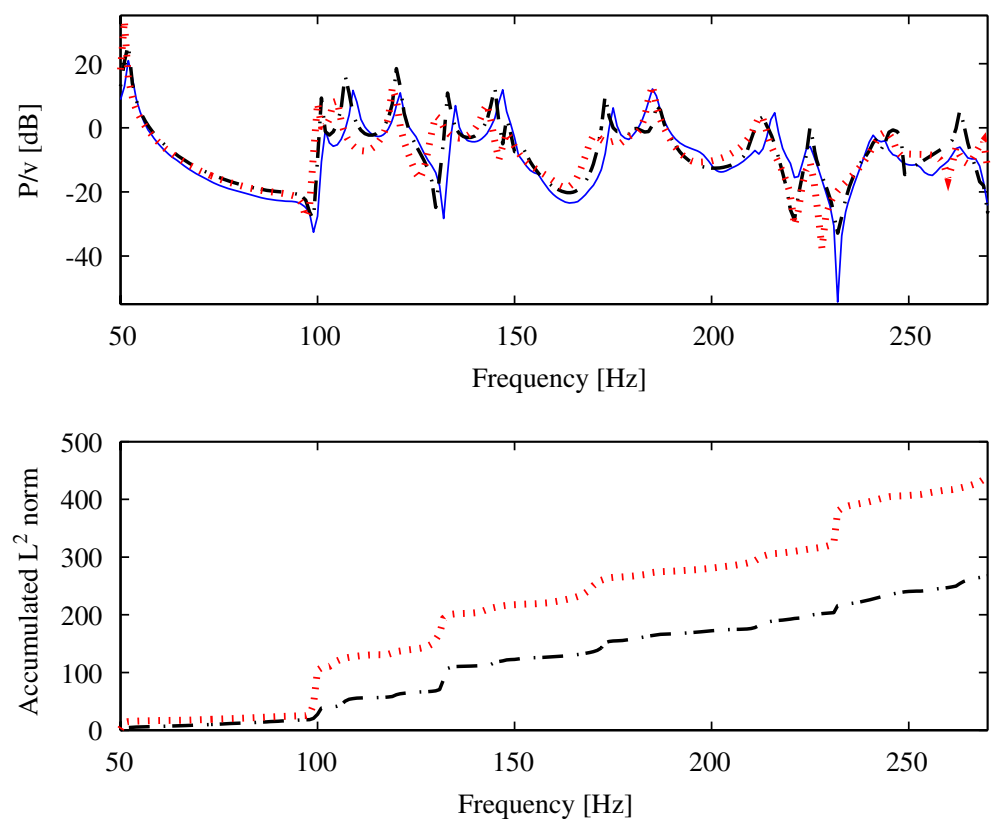

Fig. 9. Top figure: mobility amplitude from (1) measured pressure (solid line), (2) computed pressure using updated $A_{n}$ (dash-dotted line), (3) computed pressure using standard material properties (dotted line). Bottom figure: frequency accumulated error $\left(L^{2}\right.$ norm) between measured and computed mobility using (1) updated $A_{n}$ (dash-dotted line) (2) standard material properties (dotted line).

The real and imaginary parts computed with the measured material properties are slightly different from the ones calculated with the updated material characteristics. That can be partially explained by the fact that most of the measurement techniques do not take into account the tridimensional behavior of the tested material sample.

The resulting microstructural parameters of the porous media are reported in Table 3 together with the experimentally measured material properties. The shape factors cannot be measured, which explains why there is no corresponding data in Table 3. For this particular setup, the backrest and bottom cushions are made of the same absorbing material, the only difference being the thickness of the cushions. Then, during the second stage optimization process, the backrest and bottom cushions are updated simultaneously, and the intrinsic properties of the materials are imposed to be identical for both cushions.

It is also interesting to compare the FRF built using the updated admittance values to the FRF based on the initial guest that would be used if no experimental data were available and the updating process was not performed (i.e. $\Omega=1, q^{2}=1, \sigma=10^{4} \mathrm{Nm}^{-4} \mathrm{~s}$, and $S_{b}=1$ ). Fig. 9 presents the mobility at the driver's ear. The experimental mobility is compared to the mobility computed using standard material properties and using the updated values. In bottom graph of Fig. 9, the authors want to quantify the improved fitting between the measured FRF and the FRF computed based on the updated material properties versus standard material damping data. The frequency accumulated $L^{2}$ norm between the measured and computed FRF's sums the 
distance separating the curves along the frequency range. The larger the accumulated error, the worse is the curve fitting through the frequency band. Bottom graph in Fig. 9 shows that the measured versus computed mobility distance, which is accumulated through the frequency range, is much larger when using admittance coefficients calculated with standard material properties compared to updated parameters.

\section{Conclusions}

A 2-stage updating process based on the CRE technique was successfully tested and validated: it makes the CPU time slow down and allows an easy and costless comparison of different admittance models.

The CRE updating technique has been applied to the TRICARMO simplified concrete car to describe the absorbing materials of the seat of the driver. The pressure/exciting velocity ratio coming from the updating stage is compared to the experimental ratio at the driver's ear. The CRE technique, which minimizes the global error inside the whole acoustic domain, provides a quite good improvement of the computed FRF at the particular location where the updated and experimental FRF's are compared.

\section{Acknowledgments}

The authors would like to acknowledge LMS International located in Leuven (Belgium) and particularly Dr. E. Brechlin and Dr. H. Van der Auweraer for their collaboration to the present work by providing the experimental setup and data. The elaboration of the concrete car setup and all related test work were carried out by LMS International in the framework of the Flemish research project TRICARMO, which was financially supported by the Flemish Institute for the promotion of scientific and technological research in industry (IWT).

\section{References}

[1] V. Decouvreur, P. Bouillard, A. Deraemaeker, P. Ladevèze, Updating 2D acoustic models with the constitutive relation error, Journal of Sound and Vibration 278 (4/5) (2004) 773-787.

[2] D. Wilson, Simple, relaxational models for the acoustical properties of porous media, Applied Acoustics 50 (3) (1997) $171-188$.

[3] P. Ladevèze, A modelling error estimator for dynamic model updating, in: P. Ladevéze, J.T. Oden (Eds.), New Advances in Adaptive Computational Methods in Mechanics, Elsevier, 1998, pp. 135-151.

[4] J.-F. Allard, Propagation of Sound in Porous Media, Elsevier Science Publishers Ltd, 1993 ISBN 185166887.

[5] M.A. Delany, E.N. Bazley, Acoustic properties of fibrous absorbent materials, Applied Acoustics 3 (1970) $105-116$.

[6] J.-F. Allard, C. Depollier, J. Nicolas, W. Lauriks, A. Cops, Propriétés acoustiques des matériaux poreux saturés d'air et théorie de Biot, Journal d'Acoustique 3 (1990) 28-38.

[7] D. Nefske, Structural-acoustic finite element analysis of the automobile passenger compartment: a review of current practice, Journal of Sound and Vibration 80 (2) (1982) 247-266.

[8] E. Brechlin, I. Bosmans, N. Keymeulen, R. Dekkers, Experimental investigations of the vibro-acoustic behaviour of trim components in a car cabin, International Conference on Noise \& Vibration Engineering, ISMA2002, Leuven, Belgium, 2002.

[9] L. Kelders, G. Jansens, W. Lauriks, Material parameter evaluation, in: Katholieke Universiteit of Leuven Internal Report, LMS P.V. 4351, 2001, pp. 1-13. 